\title{
Time-dependent Role of Bisphosphonates on Atherosclerotic Plaque Calcification
}

Amirala Bakhshian Nik ${ }^{1}$, Hooi Hooi Ng ${ }^{1,2}$, Manuel Garcia Russo ${ }^{1}$, Francesco lacoviello ${ }^{3}$, Paul R. Shearing $^{3}$, Sergio Bertazzo ${ }^{4}$, Joshua D. Hutcheson ${ }^{1,5}$

${ }^{1}$ Department of Biomedical Engineering, Florida International University, Miami, FL 33174, USA.

${ }^{2}$ Department of Human and Molecular Genetics, Herbert Wertheim College of Medicine, Florida International University, Miami, FL 33199, USA.

${ }^{3}$ Department of Chemical Engineering, University College London, London WC1E 7JE, UK.

${ }^{4}$ Department of Medical Physics and Biomedical Engineering, University College London, London WC1E 6BT, UK.

${ }^{5}$ Biomolecular Sciences Institute, Florida International University, Miami, FL 33199, USA.

\section{Corresponding author: \\ Joshua D. Hutcheson, PhD \\ 10555 West Flagler Street, \\ Engineering Center 2600, \\ Miami, Florida 33174, USA. \\ Phone: +1-305-348-0157 \\ Email: jhutches@fiu.edu}

\begin{abstract}
Atherosclerotic plaque calcification directly contributes to the leading cause of morbidity and mortality by affecting the plaque vulnerability and rupture risk. Small microcalcifications can increase plaque stress and promote rupture, whereas large calcifications can stabilize plaques. Drugs that target bone mineralization may lead to unintended consequences on ectopic plaque calcification and cardiovascular outcomes. Bisphosphonates, common anti-osteoporotic agents, elicited unexpected cardiovascular events in clinical trials. Here, we investigated the role of bisphosphonates treatment and timing on the disruption or promotion of vascular calcification and bone mineral in a mouse model of atherosclerosis. We started the bisphosphonate treatment either before plaque formation, at early plaque formation times associated with the onset of calcification, or at late stages of plaque development. Our data indicate that long term bisphosphonate treatment (beginning prior to plaque development) leads to higher levels of plaque calcification, with a narrower mineral size distribution. When given later in plaque development, we measured a wider distribution of mineral size. These morphological alterations may associate with higher risk of plaque rupture by creating stress foci. Yet, bone mineral density positively correlated with the duration of bisphosphonate treatment.
\end{abstract}

Keywords: Atherosclerotic plaque calcification, Calcification paradox, Bisphosphonates. 


\section{Introduction}

Atherosclerosis represents the most common cause of cardiovascular disease, and atherosclerotic plaque rupture results in arterial thrombosis, leading to heart attacks and strokes $[1,2]$. Vascular smooth muscle cell migration and osteogenic differentiation promote calcification within the atherosclerotic plaque [3]. Plaque calcification occurs in $53 \%$ and $32 \%$ of American male and female atherosclerotic patients, respectively [4]. Comprehensive analyses of coronary artery calcium score have demonstrated a strong positive correlation between calcification and all-cause mortality, including cardiovascular and coronary artery disease [5]. Though overall calcium score positively predicts cardiovascular morbidity, local effects of calcification on plaque stability is determined by calcification size and morphology.

Recent clinical studies reported percent calcified plaque volume as a key factor for plaque stability [6]. Plaques with high percentages of calcification were more stable and less likely to rupture. Plaque vulnerability classically associates with low collagen content in the fibrous cap, which compromises its tensile strength [7]. In silico studies highlight the presence of destabilizing microcalcifications $(5-15 \mu \mathrm{m})$-undetectable due to resolution limits of the traditional clinical imaging modalities -in the cap of the vulnerable plaques as a determinant of their biomechanical failure [8, 9]. Large macrocalcification $(>50 \mu \mathrm{m})$ stabilizes the plaque through tissue stress factor reduction. However, microcalcifications destabilize the plaque by creating stress foci within the fibrous cap due to large mismatch in material properties between the stiff minerals and the surrounding hyperelastic extracellular matrix [8-12]. Prospective clinical data from the Multi-Ethnic Study of Atherosclerosis (MESA) cohort corroborates the biomechanical model predictions linking microcalcification with plaque rupture [12]. These data suggest that coronary artery calcification volume directly contributes with higher risk of coronary heart disease and cardiovascular events. Interestingly, calcium density inversely correlates with cardiovascular event risk [12].

Patients with bone disorders are also at high-risk for cardiovascular events [13, 14]. Unbalanced bone turnover (either high or low bone formation rate) alters the serum calcium and phosphate levels and increases the risk of vascular calcification [15]. Bisphosphonates (BiPs), the most common osteoporotic treatment option, stabilize bone mineral through their high binding affinity to hydroxyapatite and decrease bone resorption by suppressing osteoclast activity [13]. BiPs act as non-hydrolyzable analogs of inorganic pyrophosphate, a common calcification inhibitor of ectopic calcification $[13,16,17]$. However, BiPs have demonstrated potential off-target effects on cardiovascular morbidity. BiP usage correlates with higher risk of cardiovascular/cerebrovascular events and arterial fibrillation in osteoporotic patients with a history of previous cardiovascular events $[18,19]$. Retrospective clinical trials found paradoxical cardiovascular outcomes in patients taking BiPs. In women with chronic kidney disease, BiPs moderately reduced the hazard ratio of future cardiovascular events in patients with no previous cardiovascular events; yet, in patients with prior cardiovascular events, BiPs increased the hazard ratio of future events [20].

BiPs may affect vascular calcification through both direct alterations to plaque mineral and systemic effects on calcification mediators. Previous studies reported accumulation of BiPs in the arterial wall of both an atherosclerotic rabbit model and human plaques [21, 22]. Short-term BiP treatment elevated serum levels of parathyroid hormone and osteocalcin, key regulators of cardiovascular calcification [23, 24], in a female renal failure rat model [25]. BiPs prevented medial calcinosis in a male renal failure rat model; however, it was parallel to reduction in bone mass density [26]. BiPs altered the mineral microstructure in Apolipoprotein E-deficient (Apoe ${ }^{-/}$) mice fed an atherogenic diet, indicating a direct interaction between BiPs and mineral within the 
plaque [1]. Given the long term administration of BiPs for osteoporosis treatment [27], further studies are needed to assess how BiPs affect vascular calcification over the course of plaque development.

Here, we investigated the effect of BiP treatment on atherosclerotic plaque calcification when given at early, intermediate, and late stages of atherosclerosis. We began BiP administration to $\mathrm{Apoe}^{-/-}$mice at three different periods of plaque development based on previous reports [2, 28, 29]: before mineral formation (early), during plaque remodeling and mineral deposition (midterm), or after the formation of mature, calcified plaques (late stage). Our data indicate that beginning $\mathrm{BiP}$ treatments in either early or late stages promote plaque calcification; however, early $\mathrm{BiP}$ treatment resulted in narrower mineral size distribution compared to the late-stage treatment. Interestingly, beginning the $\mathrm{BiP}$ treatment during active plaque remodeling led to lower levels of plaque calcification compared to the other treatment groups. These data may provide insight into potential cardiovascular-related off-target effects of BiPs.

\section{Methods}

\subsection{In vivo Study}

The animal study was approved by the Institutional Animal Care and Use Committee (IACUC) at Florida International University under protocol 17-022 and conformed to current NIH guidelines.

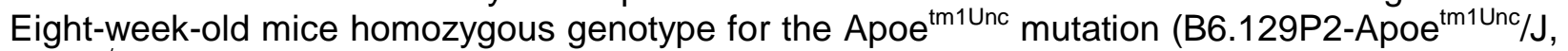
$\mathrm{ApoE}^{-/-}, n=40,20$ per biological sex) were purchased from the Jackson Laboratory (Bar Harbor, Maine, USA). After two weeks of acclimatization and feeding with a regular chow diet, the animals (10 weeks old) were fed with an atherogenic diet (ENVIGO, TD.88137 adjusted calories diet $42 \%$ from fat) for 25 weeks. Mice were randomly divided into four groups (5 males and females per treatment): Week 5, Week 10, and Week 15, in which animals received subcutaneous injections of bisphosphonate ibandronate sodium ( $2 \mathrm{mg} / \mathrm{kg} / \mathrm{mouse}$, APEXBIO, B1772) twice per week (on Mondays and Thursdays), following 5, 10, and 15 weeks of the atherogenic diet, respectively. The control group received drug vehicle, phosphate-buffered saline (PBS, 1X, Caisson Labs, PBL01). For the injections, animals were partially anesthetized using isoflurane (1\%, Patterson Veterinary, 07-893-1389, in 2 L. $\min ^{-1}$ oxygen flow). At the study end point (after 25 weeks of diet), the mice received a tail vein injection of a calcium tracer, OsteoSense 680EX (80 nmol/kg/mouse, PerkinElmer, NEV10020EX). After 48 hours, the animals were anesthetized with isoflurane $\left(1 \%\right.$, in 2 L. $\min ^{-1}$ oxygen flow) followed by retroorbital bleeding for blood collection. Mice were then immediately euthanized by laceration of the diaphragm before tissue collection. The hearts and aortas were resected and fixed in formalin $(10 \% \mathrm{w} / \mathrm{v}$, Fisher Scientific, SF100) for 2 hours. The blood samples were centrifuged at 2,000 $\times \mathrm{g}$ for 15 minutes to collect the serum.

\subsection{Calcification Morphological Quantification}

After resection and removal of excess adventitia and adipose tissues, the aortas were imaged using a near-infrared scanner (LI-COR Odyssey) to visualize the atherosclerotic plaque calcification burden. The signals were localized and quantified using edge detection algorithms in a custom MATLAB script, which quantified the total area of the calcium tracer, and normalized to the total scanned aorta area. The resected aortic roots were embedded using Tissue-Plus OCT (Fisher Scientific, 23-730-571) and cryosectioned (sagittal, $18 \mu \mathrm{m} / \mathrm{section}$ ). The serial sections were imaged using a confocal microscopy system (Eclipse Ti, Nikon). To assess total, mean, and maximum calcification area in the aortic roots, a custom image analysis script was developed in MATLAB. After filtering the background and smoothing the images, 
individual microcalcifications were identified as connected pixels in binarized images. The script reported the summation of the pixels, average area of the connected pixels (representing mean of the mineral size), and the maximum connected area (representing size of a single largest mineral) for each image as total, mean, and maximum calcification area, respectively. Connected areas smaller than 5 pixels were excluded from analyzed data.

\subsection{Serum Alkaline Phosphatase Activity and Total Cholesterol Assessment}

To assess the activity of mouse serum tissue non-specific alkaline phosphatase (TNAP), a colorimetric assay kit (BioVision, K412) was used. The samples were diluted 1:20 in assay buffer, and $80 \mu \mathrm{L}$ of each sample were mixed with $50 \mu \mathrm{L}$ of $5 \mathrm{mM}$ pNPP solution and incubated for $60 \mathrm{~min}$ at $25^{\circ} \mathrm{C}$. The colorimetric change resulting from the reaction was detected using a multi-mode reader (BioTek, Synergy HTX) to measure absorbance at $405 \mathrm{~nm}$.

To quantify the serum total cholesterol, a Wako Free Cholesterol E kit (FUJIFILM Medical Systems USA, 99902601) was used. Briefly, $10 \mu \mathrm{L}$ of each serum sample were resuspended in $1 \mathrm{~mL}$ of assay color reagent and incubated at $37^{\circ} \mathrm{C}$ for $5 \mathrm{~min}$. The colorimetric change resulting from the reaction was detected using a plate reader to measure absorbance at $600 \mathrm{~nm}$.

\subsection{X-ray Computed Tomography (X-ray CT)}

Femurs were dissected from mice and imaged directly in a Nikon XT H 225 scanner (macro-CT, Nikon Metrology, Tring, UK). The raw transmission images were reconstructed using commercial image reconstruction software package (CT Pro 3D, Nikon Metrology, Tring, UK), which employs a filtered back-projection algorithm. The scan was performed using $80 \square \mathrm{kV}$ beam energy, $70 \square \mu \mathrm{A}$ beam current, and a power of $5.6 \mathrm{~W}$. A PerkinEImer 1620 flat panel detector was used, with $200 \square \mu \mathrm{m}$ pixel size. The resulting effective pixel size was $5 \square \mu \mathrm{m}$. The exposure time per projection was $0.5 \mathrm{~s}$, and a total of 1601 projections were acquired, resulting in a scanning time of approximately 13 minutes per sample. Bone structural parameters, including thickness and volume fraction (the ratio of bone volume (BV) to total volume (TV)), for both cortical and trabecular regions were assessed using a plug-in module, BoneJ [30], in ImageJ (NIH, USA) [31].

\subsection{Statistics}

Data are presented as the mean of independent replications, and error bars represent the standard error of the mean. The reported $n$ values represent independent biological replicates. Statistical significance between groups was calculated using two-way ANOVA with mixedeffects model in GraphPad Prism 8. A p-value less than 0.05 was considered statistically significant. In the case of comparisons between two groups, the statistical significance was calculated using Welch's t-test with p-values less than 0.05 significant.

\section{Results}

3.1. BiP treatment increases atherosclerotic plaque calcification in the Apoe ${ }^{-/-}$mice fed an atherogenic diet.

Visualization and quantification of the calcium tracer OsteoSense from resected aorta revealed significantly elevated plaque calcification in all BiP-treated animals compared to the control group (Fig. 1, A), regardless of BiP regimen beginning time and sex. In male mice, early (week 5) and late (week 15) BiP treatments showed the highest plaque calcification compared to control and mid-term BiP administration (week 10), as shown in Fig. 1, B. However, female 
mice exhibited no significant difference among the BiP treated groups in terms of plaque calcification burden along the aorta (Fig. 1, C). Interestingly, female mice showed significantly higher levels of calcification compared to males when the BiP treatment began at 10 weeks of the atherogenic diet (Fig. 1, F). The level of calcification was similar between males and females in control, week 5, and week 15 groups (Fig. 1, D to G). These data demonstrate that BiP may interact with the ectopic atherosclerotic plaque calcification and increase the rate of mineral formation in a sex-dependent manner.

\subsection{BiP treatment alters the size of mineral in the atherosclerotic plaque.}

Analysis of the mineral morphologies in the aortic roots of the mice showed BiP treatment significantly increased the total calcification area in both male and female groups, compared to the control mice (Fig. 2, A, B, E, and F). Similar to the plaque calcification along the aorta, both week 5 and week 15 male groups had a significantly higher level of total calcification area, compared to the week 10 group (Fig. 2, B). However, no significant changes were observed between the female BiP-treated groups. The average calcification size was significantly bigger in early BiP-treated group (week 5) compared to the control, week 10, and week 15 groups. The mean calcification area showed no significant differences between the male control, week 10, and week 15 groups (Fig. 2, C). In female mice, the mean calcification area increased in both week 5 and week 15, compared to the control and week 10 groups. No significant changes were observed between the control and week 10 groups (Fig. 2, G). Maximum calcification area, the largest mineral detected in a plaque, increased in all BiP-treated groups compared to the control, regardless of sex. The largest calcified area in the male groups was detected in both week 5 and week 15. In female groups, early BiP treatment increased the maximum mineral size in the plaque compared to other time points, with no significant differences between week 10 and 15 (Fig. 2, H).

Comparing male and female mice, we observed no significant differences in total calcification area in the aortic roots of control, week 5, and week 15 treatment groups. However, BiP treatment beginning at 10 weeks of diet significantly increased the total calcification area in the female mice compared to the male mice (Fig. 3, panel A). The mean calcification area (mineral average size) remained unchanged between the males and females in the control, week 10, and week 15; however, in week 5 , the male mice showed a significantly elevated mean calcification area compared to the females (Fig. 3, panel B). Regardless of the time at which $\mathrm{BiP}$ treatment began, the maximum calcification area in the male groups was higher than the females; however, no significant differences were detected in the control group between males and females (Fig. 3, panel C). These data suggest that BiP treatment may interact with and alter the morphology of mineral in the plaque.

\subsection{BiP treatment does not affect serum alkaline phosphatase activity and total} cholesterol level.

We measured the level of alkaline phosphatase, an enzyme required for calcification, in the serum collected at the study endpoint. We observed no significant differences between the BiPtreated and the control groups across either sex (Fig. 4, A and B). Furthermore, the total serum cholesterol remained unchanged across BiP-treated groups compared to the controls for both male and female mice (Fig. 4, C and D).

\subsection{Bone remodeling positively correlates with the BiP treatment duration.}

Analyses of the resected bones showed dramatic increased bone volume in BiP-treated mice compared to the control group. The longer treatment with BiP (i.e., beginning the BiP treatment at the early time point) results in a higher bone volume in both cortical and trabecular areas 
(metaphyseal and epiphyseal regions), as shown in Fig. 5, panel A and B. The cortical bone thickness followed a similar trend and exhibited a positive correlation with the duration of BiP treatment. The thickness of the trabecular bone, both metaphyseal and epiphyseal regions, was significantly increased in the longest BiP-treated group (beginning of BiP regimen after 5 weeks of diet); this parameter remained unchanged for week 10 and week 15 groups compared to the control mice. Table 1 summarizes the bone microstructure parameters analyzed for each group.

\section{Discussion}

Atherosclerotic plaque calcification in Apoe ${ }^{-/-}$mice fed a chow diet begins around 45-60 weeks of age [32]; however, feeding the mice an atherogenic diet (21\% fat and $15 \%$ cholesterol) accelerates plaque calcification dramatically following 10-12 weeks of the diet [29, 33]. Apoe ${ }^{-1-}$ mice fed an atherogenic diet develop early plaque, including lipoproteins accumulation, immune system activation, and formation of cholesterol-rich foam cells between 4 to 14 weeks of feeding. Lipid cores develop following 14 to 16 weeks of the diet, and fibrous cap formation and mature plaque development occur over 18 to 20 weeks of the diet [34]. Here, we studied the role of $\mathrm{BiP}$ treatment on atherosclerotic plaque calcification in $\mathrm{Apoe}^{-/-}$mice. The animals received $\mathrm{BiP}$ treatment twice per week at three different time points: after 5 weeks of the diet, when the plaque formation has started but prior to calcification formation; after 10 weeks of diet, at the onset of plaque calcification; and after 15 weeks of diet, when the plaque is developed and contains calcified regions.

We showed that the total calcification burden was increased by both the early (starting after 5 weeks of diet and continued for 20 weeks) and late (starting after 15 weeks of diet and continued for 10 weeks) BiP treatment regimens in male mice compared to the control group that did not receive BiP treatment. The early BiP treatment led to a higher mean calcification area (bigger average mineral size) compared to the late treatment, which may stabilize the plaque by reducing the rupture risk. Given the fact that maximum calcification area was comparable between early and late BiP treatment groups, a smaller mineral size average in the week 15 group means the presence of a wider range of mineral size distribution, which may influence the plaque stability. Beginning BiP treatment at week 10 resulted in the smallest average and maximum mineral size compared to other BiP treated male mice, however not significantly different from week 15 . The female mice showed the same trends regarding average and maximum calcification size, with the smallest average mineral observed in the mice that received $\mathrm{BiP}$ treatment beginning at week 10 . However, the differences between treatment groups were less pronounced in the female mice. These data suggest that treating mice before calcification begins (week 5 ) results in the formation of larger calcifications, whereas starting BiP treatment during early (week 10) or late (week 15) stages of pro-calcific plaque remodeling leads to smaller average mineral size.

Mouse atherosclerotic plaques do not rupture, which limited our ability to perform plaque stability analyses in this study. However, our data suggest that timing of BiP intervention in relation to ongoing atherosclerotic remodeling could influence mineral morphology and thus plaque stability. Previous studies using electron microscopy revealed that timing of BiP regimen affects the morphology and topography of plaque microcalcifications (less than $5 \mu \mathrm{m}^{2}$ ) [1]; starting BiP treatment in early plaque remodeling led to bigger individual mineral aggregates with higher surface roughness, while starting at later time points reduced both mineral aggregates size and surface roughness [1]. Furthermore, mineral aggregates associated with later BiP treatment beginning showed qualitatively disorganized and loose morphologies [1]. Finite element-based predictions of these previous data suggested that the microcalcifications associated with all BiP treatment groups would reduce plaque stress compared to non-treated 
controls. The present data provide a more global analysis of calcification size and burden following BiP treatment and support the previous observation that BiP alters calcification morphology.

We showed that starting BiP treatment at early and late stages of calcific remodeling affected the total calcification area similarly in male and female mice. However, for the BiP regimen given during plaque remodeling and mineral deposition onset, total calcification area was higher in female mice compared to males. The mean calcification area (average of the mineral size) was similar across males and females in this group; yet, male mice had a larger maximum calcification area compared to the females, which suggest a wider mineral size distribution in males. These results may indicate a critical timing in respect of BiP usage. Recent clinical studies indicate a negative correlation between the cumulative BiP dosage (an indication of portion of day covered) to hazard ratio of hospitalization due to atherosclerotic cardiovascular events [35]. Female patients indicated higher hazard ratio (0.958) compared to associated male patients (0.897) [35], an effect that may explain the sex-dependent observation in our week 10 group. Furthermore, these clinical outcomes support our data that the longer BiP usage duration may lead to larger minerals with a narrow size distribution, which in silico studies suggest associate with stable plaque and lower rupture risk [8, 36].

In humans, 95\% of the serum TNAP originate from bone and liver [37], and unbalanced bone turnover, aging, and chronic kidney disease correlate with abnormal elevated serum TNAP [38, 39]. Serum TNAP increases in osteoporotic postmenopausal women compared to postmenopausal women without osteoporosis [38]. BiPs lowered serum TNAP in osteoporotic postmenopausal women and men with heterotopic ossification [38, 40, 41]. Our data showed unchanged TNAP in serum across the treated and untreated groups. Thus, the observed changes in plaque calcification in all treated groups appears to be independent of systemic TNAP changes.

Clinical trials reported serum LDL reduction in osteoporotic females treated with BiPs; however, the level of high-density lipoprotein and lactate dehydrogenase remained unchanged [42-44]. In the present study, serum total cholesterol remained unchanged in all animals, regardless of the $\mathrm{BiP}$ treatment. Our data indicate that the observed calcification differences by BiP treatment are not due to effects on lipid metabolism in Apoe $^{-/-}$mice.

The outcomes from bone microstructure analyses demonstrate the substantial effect of BiP treatment on bone remodeling. These results reveal a positive correlation between BiP regimen treatment duration and the bone volume and thickness in cortical and trabecular areas. We showed a positive correlation between bone remodeling and increased plaque calcification for early BiP treatment in Apoe ${ }^{-/-}$mice. Long term BiP treatment led to increased mineralization in both bone and atherosclerotic plaque. However, for later time points, beginning BiP treatment after 10 and 15 weeks of diet, the data demonstrate a negative correlation between bone remodeling and plaque calcification. Both cortical and trabecular bone volumes are significantly higher in the week 10 group, compared to week 15, while plaque calcification for the latter is significantly higher compared to former. Previous studies reported that BiP may imitate the effects of pyrophosphate, a mineralization inhibitor in the cardiovascular system; however, our data did not support the inhibitory role of $\mathrm{BiP}$ to reduce cardiovascular calcification compared to non-treated controls. Importantly, the choice of animal models or patients in clinical trials may affect the BiP outcomes; atherosclerotic plaque calcification represents a complex process in which several cell types, including VSMCs and macrophages [45, 46], are involved, thus, the $\mathrm{BiP}$ treatment may affect multiple aspects of plaque progression and calcification. 


\section{Conclusion}

Atherosclerotic plaque calcification represents a significant predictor of lesion vulnerability. Patients with bone disorders are prone to develop ectopic cardiovascular calcification. Clinical trials correlated BiPs, a common osteoporotic pharmaceutical family, with contradicting cardiovascular outcomes. Here, we demonstrated the importance of treatment timing in BiPinduced mineral disruption or promotion. We indicated that BiP can alter key morphological features of the microcalcifications within the atherosclerotic plaque of $\mathrm{Apoe}^{-/-}$mice, which may determine the risk of plaque rupture. Early beginning of BiP regimen, i.e., before calcification initiates, increased the total calcification in both males and females; however, the treatment led to narrower mineral size distribution. Later regimen timings, either after mineralization starts or when the plaque is developed, result in wider mineral size distribution, which correlates with plaque destabilization and a higher risk of rupture. Interestingly, early BiP regimen elevated mineralization in both bone and atherosclerotic plaque. However, BiP regimen correlates negatively with bone mineralization and plaque calcification if given after the onset of plaque mineralization.

\section{Funding}

This research was funded by the American Heart Association, grant number 17SDG633670259, awarded to J.D.H. A.B.N. was supported by the Florida International University Graduate School Dissertation Year Fellowship. H.H.N was supported by the American Heart Association Postdoctoral Fellowship (19POST34380255) and the 2021 "Stop Heart Disease" Researcher of the Year Award from Florida Heart Research Foundation.

\section{Author Contributions}

The author contributions are as following: Conceptualization, A.B.N. and J.D.H.; Methodology, S.B. and J.D.H.; Software, A.B.N., M.G.R. and J.D.H.; Validation, A.B.N., M.G.R., F.I., P.R.S., S.B. and J.D.H.; Formal Analysis, A.B.N., F.I., P.R.S. and J.D.H.; Investigation, A.B.N., H.H.N., F.I. and P.R.S.; Resources, H.H.N., F.I. and J.D.H.; Data Curation, A.B.N. and M.G.R.; Writing Original Draft Preparation, A.B.N.; Writing - Review \& Editing, H.H.N., M.G.R., F.I., P.R.S., S.B. and J.D.H.; Visualization, A.B.N.; Supervision, J.D.H.; Project Administration, J.D.H.; Funding Acquisition, J.D.H. 


\section{References}

[1] J. L. Ruiz et al., "Nanoanalytical analysis of bisphosphonate-driven alterations of microcalcifications using a 3D hydrogel system and in vivo mouse model," Proceedings of the National Academy of Sciences, vol. 118, no. 14, 2021.

[2] J. D. Hutcheson et al., "Genesis and growth of extracellular-vesicle-derived microcalcification in atherosclerotic plaques," Nature materials, vol. 15, no. 3, pp. 335-343, 2016.

[3] J. F. Bentzon, F. Otsuka, R. Virmani, and E. Falk, "Mechanisms of plaque formation and rupture," Circulation research, vol. 114, no. 12, pp. 1852-1866, 2014.

[4] S. S. Virani et al., "Heart disease and stroke statistics-2021 update: a report from the American Heart Association," Circulation, vol. 143, no. 8, pp. e254-e743, 2021.

[5] A. W. Peng et al., "Long-term all-cause and cause-specific mortality in asymptomatic patients with CAC $\geq 1,000$ : results from the CAC Consortium," Cardiovascular Imaging, vol. 13, no. 1_Part_1, pp. 83-93, 2020.

[6] H.-Y. Jin et al., "The relationship between coronary calcification and the natural history of coronary artery disease," Cardiovascular Imaging, vol. 14, no. 1, pp. 233-242, 2021.

[7] P. Libby, "Collagenases and cracks in the plaque," The Journal of clinical investigation, vol. 123, no. 8, pp. 3201-3203, 2013.

[8] A. Kelly-Arnold, N. Maldonado, D. Laudier, E. Aikawa, L. Cardoso, and S. Weinbaum, "Revised microcalcification hypothesis for fibrous cap rupture in human coronary arteries," Proceedings of the National Academy of Sciences, vol. 110, no. 26, pp. 10741-10746, 2013.

[9] N. Maldonado, A. Kelly-Arnold, L. Cardoso, and S. Weinbaum, "The explosive growth of small voids in vulnerable cap rupture; cavitation and interfacial debonding," Journal of biomechanics, vol. 46, no. 2, pp. 396-401, 2013.

[10] J. L. Ruiz, J. D. Hutcheson, and E. Aikawa, "Cardiovascular calcification: current controversies and novel concepts," Cardiovascular Pathology, vol. 24, no. 4, pp. 207-212, 2015.

[11] Y. Wang, M. T. Osborne, B. Tung, M. Li, and Y. Li, "Imaging cardiovascular calcification," Journal of the American Heart Association, vol. 7, no. 13, p. e008564, 2018.

[12] M. H. Criqui et al., "Calcium density of coronary artery plaque and risk of incident cardiovascular events," Jama, vol. 311, no. 3, pp. 271-278, 2014.

[13] R. R. Recker, E. M. Lewiecki, P. D. Miller, and J. Reiffel, "Safety of bisphosphonates in the treatment of osteoporosis," The American journal of medicine, vol. 122, no. 2, pp. S22-S32, 2009.

[14] C. E. Lampropoulos, I. Papaioannou, and D. P. D'cruz, "Osteoporosis-a risk factor for cardiovascular disease?," Nature Reviews Rheumatology, vol. 8, no. 10, p. 587, 2012.

[15] E. G. Neven, M. E. De Broe, and P. C. d'Haese, "Prevention of vascular calcification with bisphosphonates without affecting bone mineralization: a new challenge?," Kidney international, vol. 75, no. 6, pp. 580-582, 2009.

[16] G. A. Rodan and H. A. Fleisch, "Bisphosphonates: mechanisms of action," The Journal of clinical investigation, vol. 97, no. 12, pp. 2692-2696, 1996.

[17] A. Bakhshian Nik, J. D. Hutcheson, and E. Aikawa, "Extracellular vesicles as mediators of cardiovascular calcification," Frontiers in cardiovascular medicine, vol. 4, p. 78, 2017. 
[18] U. Kirchmayer et al., "Bisphosphonates and cardiovascular risk in elderly patients with previous cardiovascular disease: a population-based nested case-control study in Italy," Therapeutic advances in drug safety, vol. 10, p. 2042098619838138, 2019.

[19] S.-T. Wu, J.-F. Chen, and C.-J. Tsai, "The impact of bisphosphonates on mortality and cardiovascular risk among osteoporosis patients after cardiovascular disease," Journal of the Formosan Medical Association, vol. 120, no. 11, pp. 1957-1966, 2021.

[20] R. M. Perkins, H. L. Kirchner, K. Matsushita, I. D. Bucaloiu, E. Norfolk, and J. E. Hartle, "Bisphosphonates and Mortality in Women with CKD and the Presence or Absence of Cardiovascular Disease," Clinical Journal of the American Society of Nephrology, vol. 9, no. 5, pp. 874-880, 2014.

[21] R. Ylitalo, J. Monkkonen, A. Urtti, and P. Ylitalo, "Accumulation of bisphosphonates in the aorta and some other tissues of healthy and atherosclerotic rabbits," Journal of Laboratory and Clinical Medicine, vol. 127, no. 2, pp. 200-206, 1996.

[22] R. Ylitalo et al., "Accumulation of bisphosphonates in human artery and their effects on human and rat arterial function in vitro," Pharmacology \& toxicology, vol. 83, no. 3, pp. 125-131, 1998.

[23] C. Goettsch, H. Iwata, and E. Aikawa, "Parathyroid hormone: critical bridge between bone metabolism and cardiovascular disease," ed: Am Heart Assoc, 2014.

[24] A. Tacey, T. Qaradakhi, T. Brennan-Speranza, A. Hayes, A. Zulli, and I. Levinger, "Potential role for osteocalcin in the development of atherosclerosis and blood vessel disease," Nutrients, vol. 10, no. 10, p. 1426, 2018.

[25] Z. Geng, M. Monier-Faugere, F. Bauss, and H. Malluche, "Short-term administration of the bisphosphonate ibandronate increases bone volume and prevents hyperparathyroid bone changes in mild experimental renal failure," Clinical nephrology, vol. 54, no. 1, pp. 45-53, 2000.

[26] K. A. Lomashvili, M.-C. Monier-Faugere, X. Wang, H. H. Malluche, and W. C. O'neill, "Effect of bisphosphonates on vascular calcification and bone metabolism in experimental renal failure," Kidney international, vol. 75, no. 6, pp. 617-625, 2009.

[27] M. Pirmohamed, "Commentary—bisphosphonates and calcium homoeostasis," Postgraduate medical journal, vol. 76, no. 897, pp. 418-419, 2000.

[28] E. Aikawa et al., "Osteogenesis associates with inflammation in early-stage atherosclerosis evaluated by molecular imaging in vivo," Circulation, vol. 116, no. 24, pp. 2841-2850, 2007.

[29] M. G. MacAskill et al., "Characterisation of an atherosclerotic micro-calcification model using ApoE-/- mice and PET/CT," International Journal of Cardiology. Heart \& Vasculature, vol. 31, 2020.

[30] R. Domander, A. A. Felder, and M. Doube, "BoneJ2-refactoring established research software," Wellcome Open Research, vol. 6, 2021.

[31] L. Steiner, A. Synek, and D. H. Pahr, "Comparison of different microCT-based morphology assessment tools using human trabecular bone," Bone reports, vol. 12, p. 100261, 2020.

[32] M. Rattazzi et al., "Calcification of advanced atherosclerotic lesions in the innominate arteries of ApoE-deficient mice: potential role of chondrocyte-like cells," Arteriosclerosis, thrombosis, and vascular biology, vol. 25, no. 7, pp. 1420-1425, 2005.

[33] B. E. Veseli et al., "Animal models of atherosclerosis," European journal of pharmacology, vol. 816, pp. 3-13, 2017.

[34] S. C. Whitman, "A practical approach to using mice in atherosclerosis research," The Clinical Biochemist Reviews, vol. 25, no. 1, p. 81, 2004.

[35] M. Casula et al., "Association between the cumulative exposure to bisphosphonates and hospitalization for atherosclerotic cardiovascular events: A population-based study," Atherosclerosis, vol. 301, pp. 1-7, 2020.

[36] N. Maldonado et al., "A mechanistic analysis of the role of microcalcifications in atherosclerotic plaque stability: potential implications for plaque rupture," American Journal of Physiology-Heart and Circulatory Physiology, vol. 303, no. 5, pp. H619-H628, 2012.

[37] C. H. Linder, U. H. Englund, S. Narisawa, J. L. Millán, and P. Magnusson, "Isozyme profile and tissue-origin of alkaline phosphatases in mouse serum," Bone, vol. 53, no. 2, pp. 399-408, 2013.

[38] K. Mukaiyama, M. Kamimura, S. Uchiyama, S. Ikegami, Y. Nakamura, and H. Kato, "Elevation of serum alkaline phosphatase (ALP) level in postmenopausal women is caused by high bone turnover," Aging clinical and experimental research, vol. 27, no. 4, pp. 413-418, 2015. 
[39] C. P. Kovesdy, V. Ureche, J. L. Lu, and K. Kalantar-Zadeh, "Outcome predictability of serum alkaline phosphatase in men with pre-dialysis CKD," Nephrology Dialysis Transplantation, vol. 25, no. 9, pp. 3003-3011, 2010.

[40] A. Ploumis et al., "Association between alendronate, serum alkaline phosphatase level, and heterotopic ossification in individuals with spinal cord injury," The journal of spinal cord medicine, vol. 38, no. 2, pp. 193-198, 2015.

[41] B. Y. Klein, H. Ben-Bassat, E. Breuer, V. Solomon, and G. Golomb, "Structurally different bisphosphonates exert opposing effects on alkaline phosphatase and mineralization in marrow osteoprogenitors," Journal of cellular biochemistry, vol. 68, no. 2, pp. 186-194, 1998.

[42] E. Guney, G. Kisakol, A. G. Ozgen, C. Yilmaz, and T. Kabalak, "Effects of bisphosphonates on lipid metabolism," Neuroendocrinology Letters, vol. 29, no. 2, pp. 252-255, 2008.

[43] S. Adami, V. Braga, G. Guidi, D. Gatti, D. Gerardi, and E. Fracassi, "Chronic intravenous aminobisphosphonate therapy increases high-density lipoprotein cholesterol and decreases low-density lipoprotein cholesterol," Journal of bone and mineral research, vol. 15, no. 3, pp. 599604, 2000.

[44] M. P. Whyte, L. G. Kempa, W. H. McAlister, F. Zhang, S. Mumm, and D. Wenkert, "Elevated serum lactate dehydrogenase isoenzymes and aspartate transaminase distinguish Albers-Schönberg disease (chloride channel 7 deficiency osteopetrosis) among the sclerosing bone disorders," Journal of Bone and Mineral Research, vol. 25, no. 11, pp. 2515-2526, 2010.

[45] S. E. New et al., "Macrophage-derived matrix vesicles: an alternative novel mechanism for microcalcification in atherosclerotic plaques," Circulation research, vol. 113, no. 1, pp. 72-77, 2013.

[46] M. A. Rogers, M. Aikawa, and E. Aikawa, "Macrophage heterogeneity complicates reversal of calcification in cardiovascular tissues," ed: Am Heart Assoc, 2017. 
A

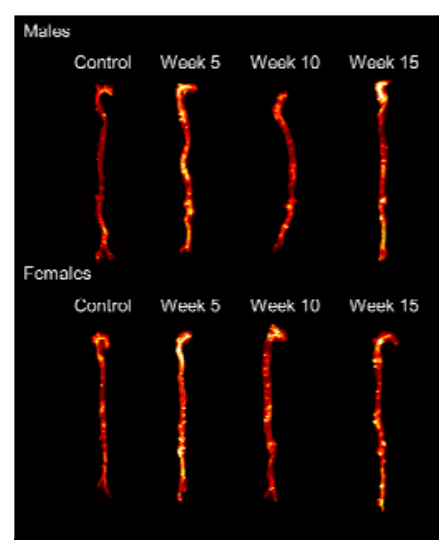

D

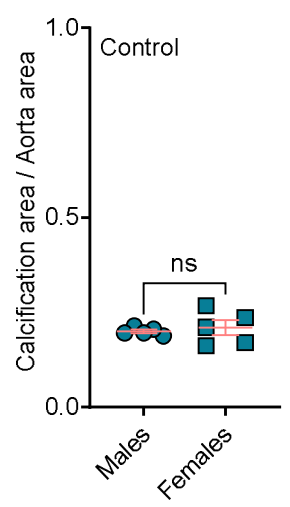

B

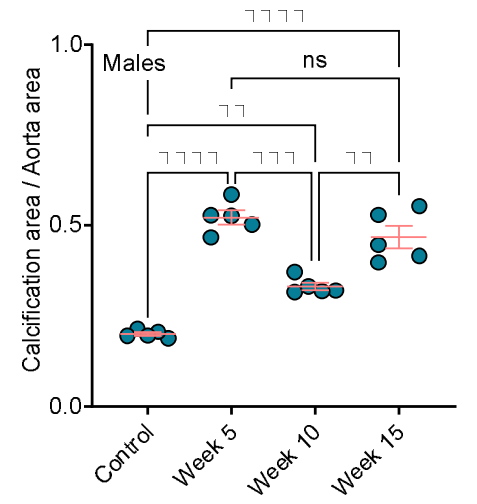

E

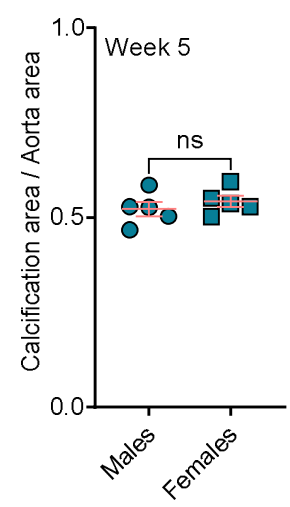

$\mathbf{F}$

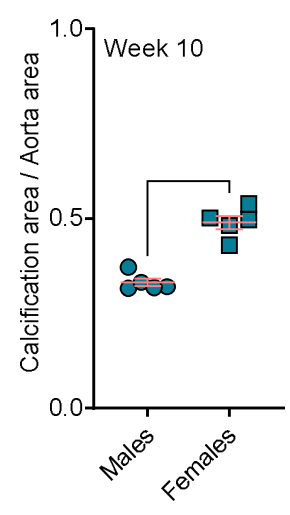

C

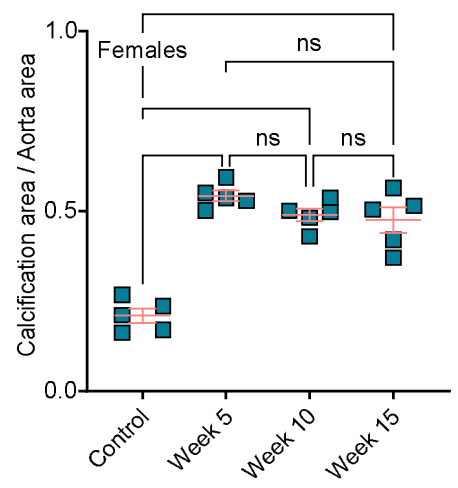

G

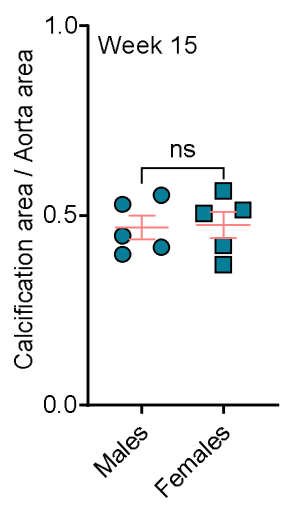

Figure 1. BiP treatment increases the atherosclerotic plaque calcification in Apoe $^{-/-}$mice fed an atherogenic diet. $(A)$ visualization of the calcium burden using a near-infrared calcium tracer, OsteoSense; ( $B$ and $C$ ) quantification of OsteoSense signal and correlation to total calcification in male and female mice, respectively; ( $D$ to $G$ ) comparison of total calcification between male and female mice in each group. ${ }^{* *} P \leq 0.01$, ${ }^{* *} P \leq 0.001$, and ${ }^{* * *} P \leq 0.0001$, two-way ANOVA with Tukey's post-hoc test for comparison between multiple groups, and Welch's t-test for comparison between two groups. 
A

$\mathbf{E}$

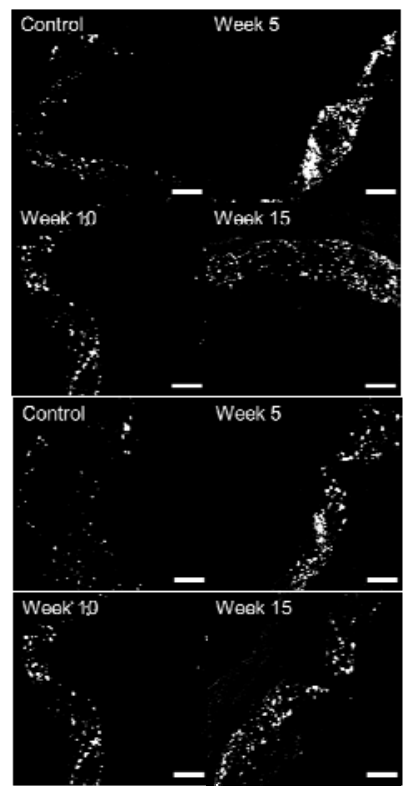

B
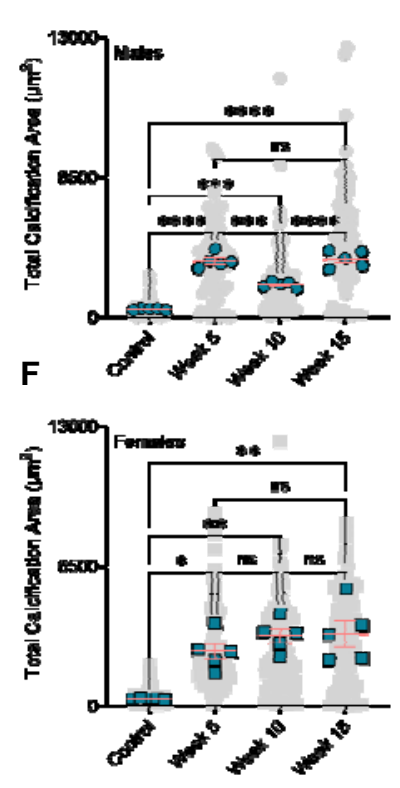

C
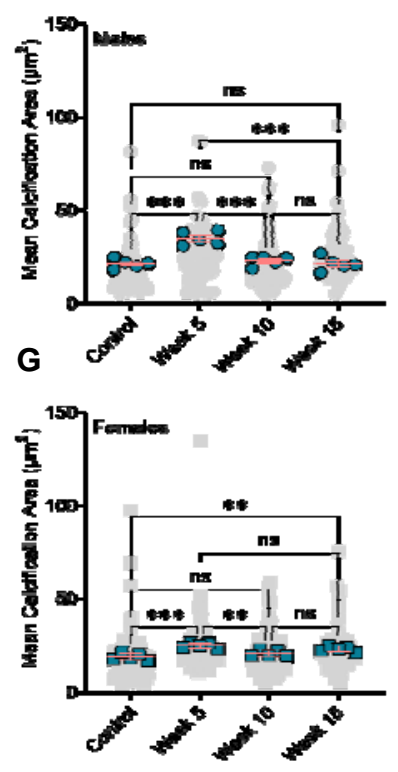

D
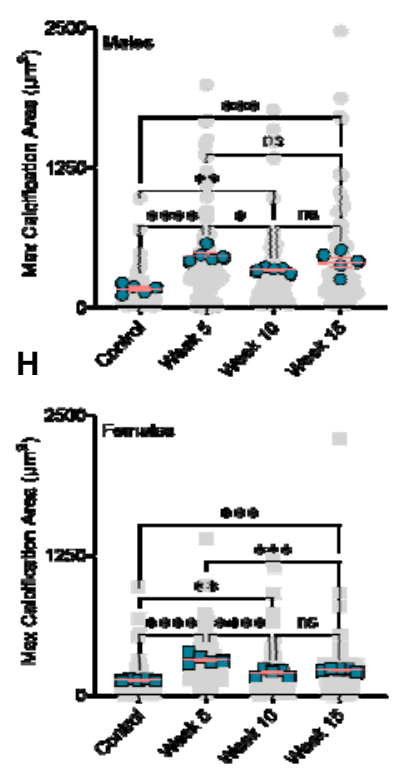

Figure 2. BiP treatment alters the micromorphology of the minerals in the atherosclerotic plaque. (A) visualization of the minerals in the atherosclerotic plaques of male mice (20X, scale bar $100 \mu \mathrm{m}$ ); (B) total plaque calcification in male mice; (C) mean calcification area (mean of mineral size) in male animals; (D) maximum calcification area in male mice; (E) visualization of the minerals in the atherosclerotic plaques of female mice $(20 \mathrm{X}$, scale bar $100 \mu \mathrm{m})$; $(\mathrm{F})$ total plaque calcification in female mice; $(G)$ mean calcification area (mean of mineral size) in female animals; $(\mathrm{H})$ maximum calcification area in female mice. ${ }^{\star} \mathrm{P}<0.05,{ }^{* \star} \mathrm{P} \leq 0.01,{ }^{\star \star *} \mathrm{P} \leq 0.001$, and ${ }^{* * \star *} \mathrm{P} \leq 0.0001$, two-way ANOVA with Tukey's post-hoc test. 

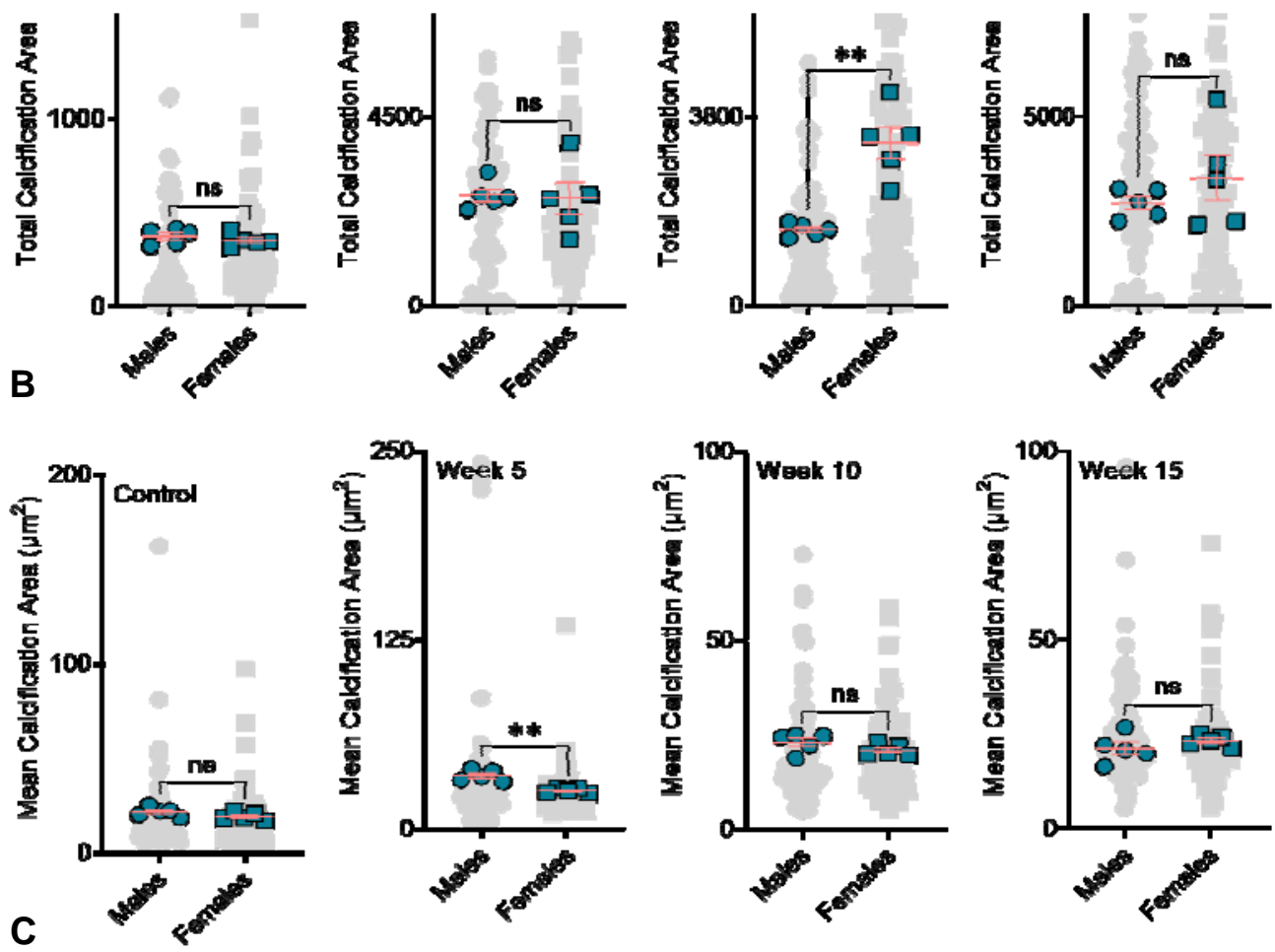

Figu
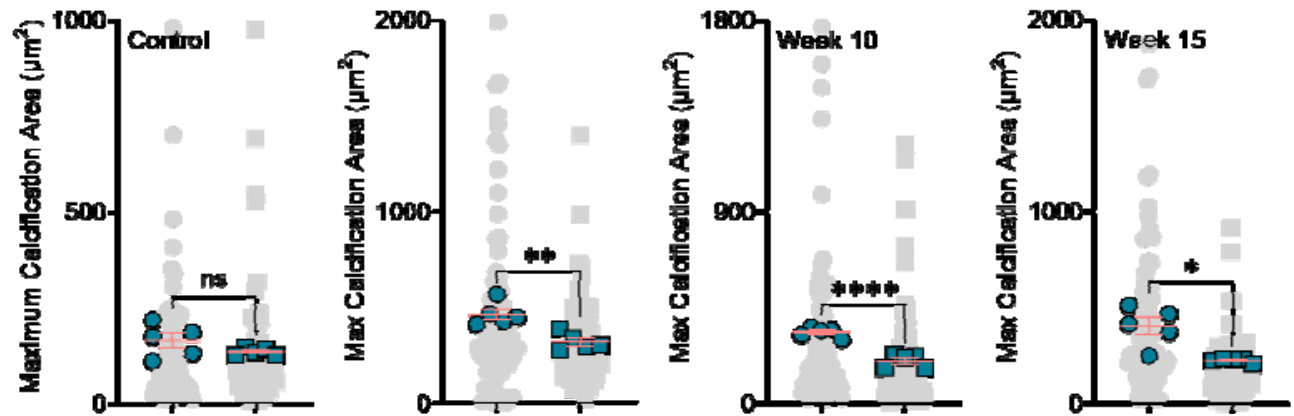

mou:

mice

(C) $\mathrm{C}$

0.01 , 
A

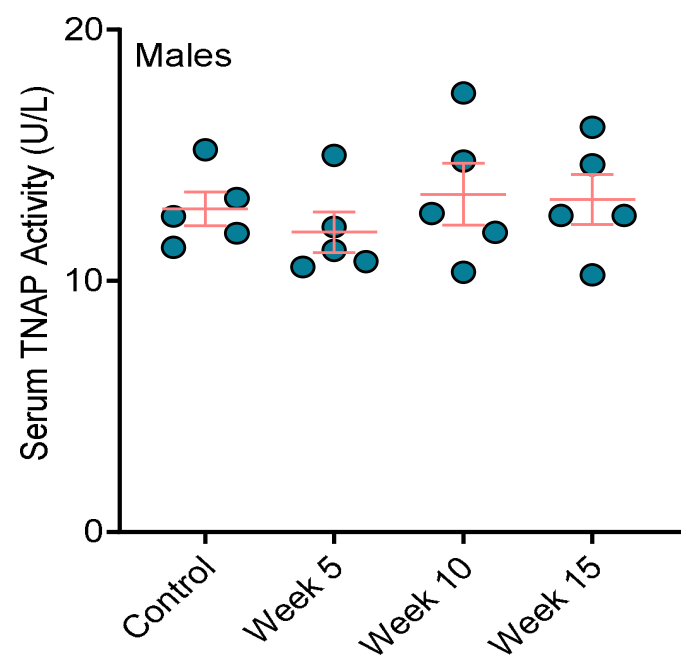

C

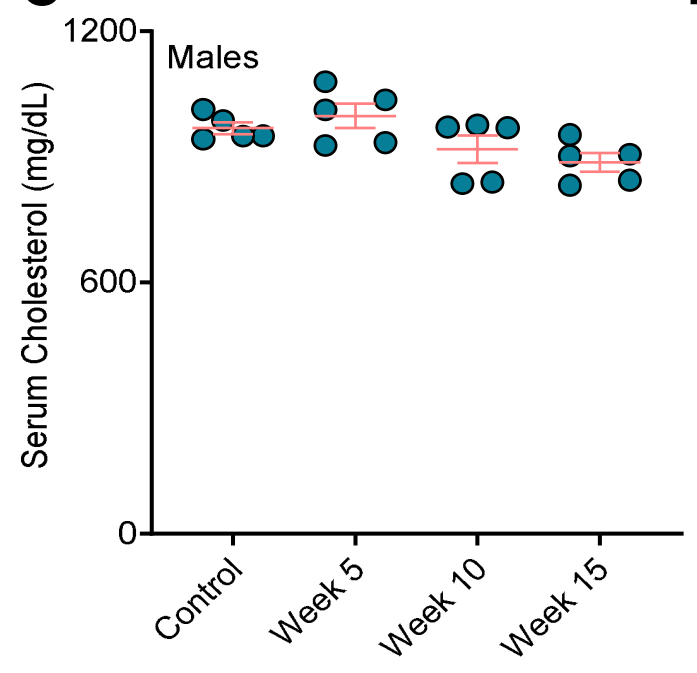

B

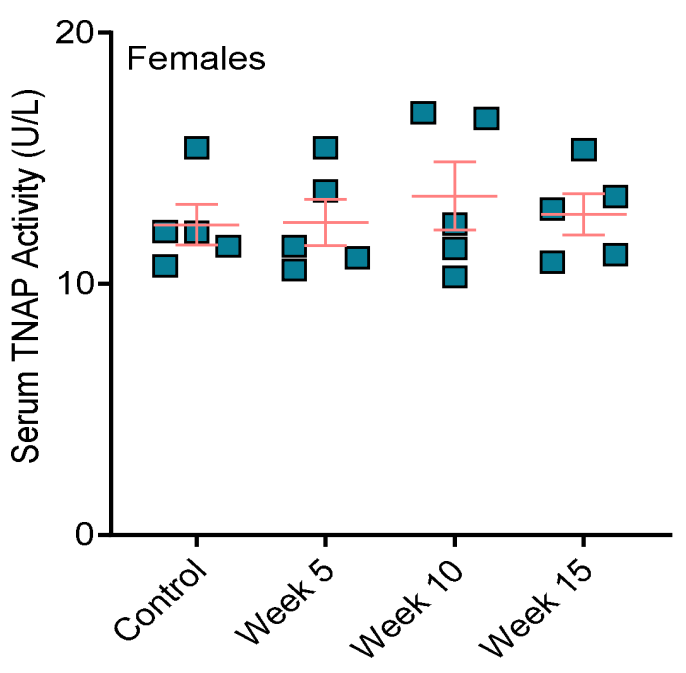

D

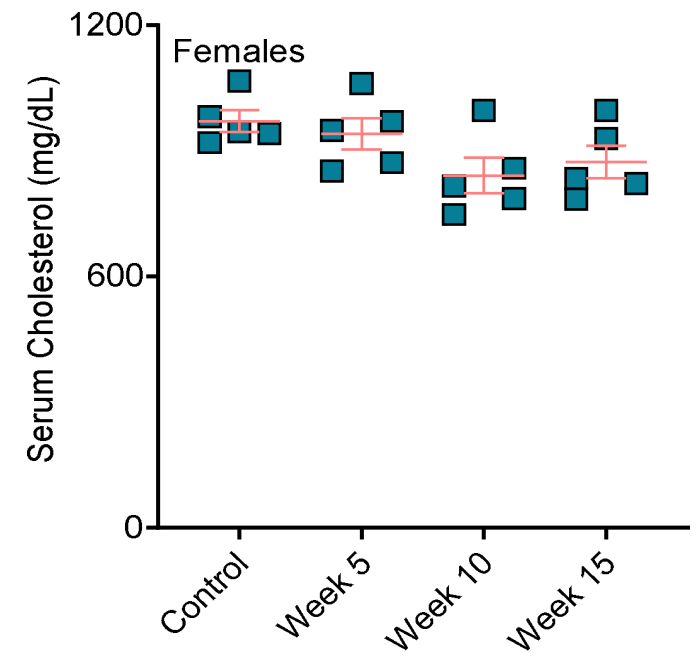

Figure 4. BiP treatment does not affect the serum TNAP activity and total cholesterol in mouse model of atherosclerotic plaque calcification. Serum TNAP activity in (A) male mice and (B) female mice; serum total cholesterol in (C) male mice and (D) female mice. No statistically significance observed across the groups, two-way ANOVA with Tukey's post-hoc test. 
Figure 5.

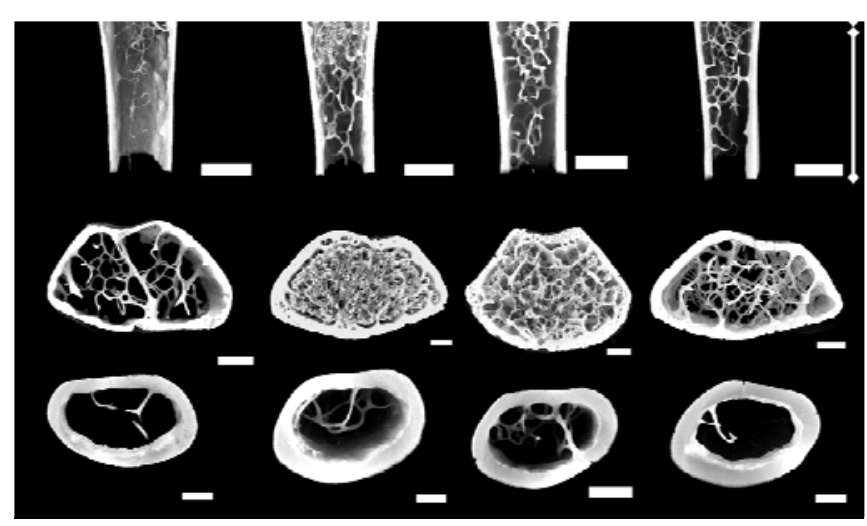

Midshaft

Ct. Bone

B
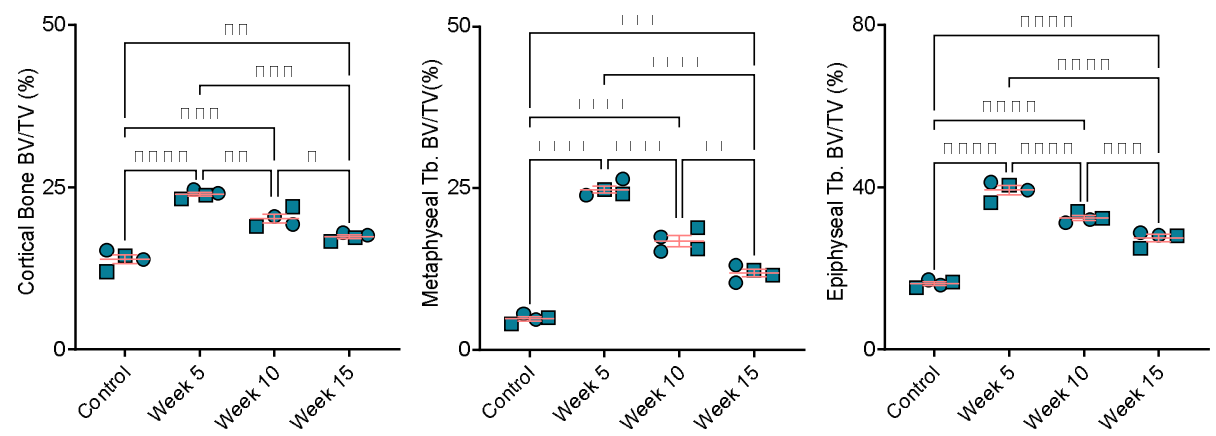

C
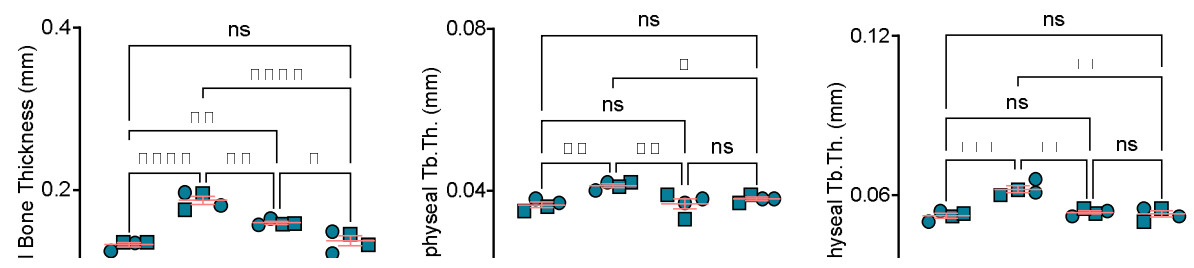

reconstruc

Panel (B)

Panel (C)

and ${ }^{* * *} \mathrm{P}$

(a).

Table 1. Detailed bone structural parameters.

\begin{tabular}{lcccc}
\hline Parameter & $\begin{array}{c}\text { Control } \\
\boldsymbol{n}=\mathbf{4}\end{array}$ & $\begin{array}{c}\text { Week 5 } \\
\boldsymbol{n}=\mathbf{4}\end{array}$ & $\begin{array}{c}\text { Week 10 } \\
\boldsymbol{n}=\mathbf{4}\end{array}$ & $\begin{array}{c}\text { Week 15 } \\
\boldsymbol{n}=\mathbf{4}\end{array}$ \\
\hline Distal femur & & & & \\
Epiphyseal Trabecular Bone & & & \\
BV/TV $(\%)$ & $16.22 \pm 0.37$ & $39.3 \pm 0.96$ & $32.4 \pm 0.5$ & $27.48 \pm 0.76$ \\
Tb.N $\left(\mathrm{mm}^{-1}\right)$ & $7.63 \pm 1.11$ & $12.86 \pm 0.11$ & $12.82 \pm 0.32$ & $12.05 \pm 0.34$
\end{tabular}




\begin{tabular}{|c|c|c|c|c|}
\hline Tb.Th (mm) & $0.052 \pm 0.001$ & $0.062 \pm 0.001$ & $0.053 \pm 0.001$ & $0.053 \pm 0.001$ \\
\hline Tb.Sp (mm) & $0.59 \pm 0.06$ & $0.41 \pm 0.02$ & $0.42 \pm 0.02$ & $0.43 \pm 0.02$ \\
\hline $\begin{array}{l}\mathrm{BS} / \mathrm{BV} \\
\left(\mathrm{mm}^{2} / \mathrm{mm}^{3}\right)\end{array}$ & $0.065 \pm 0.002$ & $0.059 \pm 0.003$ & $0.057 \pm 0.003$ & $0.062 \pm 0.003$ \\
\hline EF & $0.017 \pm 0.003$ & $0.033 \pm 0.005$ & $0.034 \pm 0.009$ & $0.016 \pm 0.003$ \\
\hline $\mathrm{EF}_{\max }$ & $0.885 \pm 0.004$ & $0.902 \pm 0.005$ & $0.887 \pm 0.004$ & $0.88 \pm 0.009$ \\
\hline $\begin{array}{l}\mathrm{EF}_{\min } \\
\mathrm{DA}\end{array}$ & $\begin{array}{c}-0.82 \pm 0.003 \\
1.43 \pm 0.05\end{array}$ & $\begin{array}{c}-0.825 \pm 0.008 \\
1.39 \pm 0.04\end{array}$ & $\begin{array}{c}-0.812 \pm 0.007 \\
1.42 \pm 0.03\end{array}$ & $\begin{array}{c}-0.81 \pm 0.009 \\
1.4 \pm 0.07\end{array}$ \\
\hline \multicolumn{5}{|c|}{ Metaphyseal Trabecular Bone } \\
\hline $\mathrm{BV} / \mathrm{TV}(\%)$ & $4.82 \pm 0.29$ & $24.8 \pm 0.5$ & $16.8 \pm 0.75$ & $11.86 \pm 0.5$ \\
\hline Tb.N $\left(\mathrm{mm}^{-1}\right)$ & $4.1 \pm 0.3$ & $13.1 \pm 0.09$ & $11.02 \pm 0.07$ & $7.62 \pm 0.49$ \\
\hline Tb.Th (mm) & $0.036 \pm 0.001$ & $0.041 \pm 0.001$ & $0.037 \pm 0.001$ & $0.038 \pm 0.001$ \\
\hline Tb.Sp (mm) & $0.56 \pm 0.094$ & $0.29 \pm 0.04$ & $0.27 \pm 0.015$ & $0.44 \pm 0.022$ \\
\hline $\begin{array}{l}\text { BS/BV } \\
\left(\mathrm{mm}^{2} / \mathrm{mm}^{3}\right)\end{array}$ & $0.092 \pm 0.003$ & $0.085 \pm 0.001$ & $0.092 \pm 0.003$ & $0.092 \pm 0.01$ \\
\hline $\mathrm{EF}$ & $0.12 \pm 0.02$ & $0.07 \pm 0.005$ & $0.086 \pm 0.017$ & $0.07 \pm 0.03$ \\
\hline $\mathrm{EF}_{\max }$ & $0.835 \pm 0.019$ & $0.87 \pm 0.002$ & $0.86 \pm 0.012$ & $0.855 \pm 0.03$ \\
\hline $\mathrm{EF}_{\min }$ & $-0.67 \pm 0.036$ & $-0.765 \pm 0.013$ & $-0.71 \pm 0.022$ & $-0.73 \pm 0.05$ \\
\hline $\mathrm{DA}$ & $1.77 \pm 0.14$ & $1.6 \pm 0.15$ & $1.522 \pm 0.09$ & $1.78 \pm 0.09$ \\
\hline \multicolumn{5}{|l|}{$\begin{array}{l}\text { Femoral midshaft } \\
\text { Cortical Bone }\end{array}$} \\
\hline Mean Ct.Th (mm) & $0.133 \pm 0.002$ & $0.187 \pm 0.004$ & $0.159 \pm 0.001$ & $0.137 \pm 0.005$ \\
\hline $\mathrm{BV} / \mathrm{TV}(\%)$ & $13.9 \pm 0.48$ & $23.9 \pm 0.25$ & $20.2 \pm 0.6$ & $17.3 \pm 0.24$ \\
\hline Ps.Pm (mm) & $5.65 \pm 0.11$ & $5.37 \pm 0.2$ & $5.35 \pm 0.22$ & $5.33 \pm 0.18$ \\
\hline Ec.Pm (mm) & $4.75 \pm 0.11$ & $4.2 \pm 0.18$ & $4.15 \pm 0.2$ & $4.17 \pm 0.21$ \\
\hline $\mathrm{J}\left(\mathrm{mm}^{4}\right)$ & $0.49 \pm 0.03$ & $0.455 \pm 0.04$ & $0.417 \pm 0.007$ & $0.5 \pm 0.1$ \\
\hline
\end{tabular}

Data were combination of males and females, and collected using X-ray Computed Tomography of distal femur and femoral midshaft. Values represent the mean \pm SEM.

$\mathrm{BV} / \mathrm{TV}=$ Bone Volume/Total Volume; Tb. $\mathrm{N}=$ Trabecular Number; Tb. Th = Trabecular Thickness; Tb.Sp = Trabecular Separation; BS/BV = Specific Bone Surface: Bone Surface Area/Bone Volume; EF = Ellipsoidal Factor; DA = Degree of Anisotropy; Ct.Th = Cortical Thickness; Ps.Pm = Periosteal Perimeter; Ec.Pm = Endocortical Perimeter; $\mathrm{J}=$ Polar Moment of Inertia. 